IJMMS 29:12 (2002) 719-726

PII. S0161171202012863

http://ijmms.hindawi.com

(c) Hindawi Publishing Corp.

\title{
RICCI CURVATURE OF SUBMANIFOLDS \\ IN KENMOTSU SPACE FORMS
}

\author{
KADRI ARSLAN, RIDVAN EZENTAS, ION MIHAI, \\ CENGIZHAN MURATHAN, and CIHAN ÖZGÜR
}

Received 20 April 2001 and in revised form 21 August 2001

\begin{abstract}
In 1999, Chen established a sharp relationship between the Ricci curvature and the squared mean curvature for a submanifold in a Riemannian space form with arbitrary codimension. Similar problems for submanifolds in complex space forms were studied by Matsumoto et al. In this paper, we obtain sharp relationships between the Ricci curvature and the squared mean curvature for submanifolds in Kenmotsu space forms.
\end{abstract}

2000 Mathematics Subject Classification: 53C25, 53C40.

1. Preliminaries. Let $(\tilde{M},<,>)$ be a Hermitian manifold and denote by $J$ the canonical almost complex structure on $\tilde{M}$. According to the behavior of the tangent bundle $T M$ with respect to the action of $J$, we may distinguish two special classes of submanifolds $M$ in $\tilde{M}$ :

(a) complex submanifolds, that is, $J\left(T_{p} M\right)=T_{p} M$, for all $p \in M$.

(b) totally real submanifolds, that is, $J\left(T_{p} M\right) \subset T_{p}^{\perp} M$, for all $p \in M$, where $T_{p} M$ (resp., $T_{p}^{\perp} M$ ) is the tangent (resp., the normal) vector space of $M$ at $p$. Such submanifolds were defined and studied by Chen and Ogiue [4].

On the other hand, Yano and Ishihara [8] considered a submanifold $M$ whose tangent bundle $T M$ splits into a complex subbundle $\mathscr{D}$ and a totally real subbundle $\mathscr{D}^{\perp}$. Later, such a submanifold was called a $C R$-submanifold [1, 2]. Blair and Chen [1] proved that a $C R$-submanifold of a locally conformal Kaehler manifold is a CauchyRiemann manifold in the sense of Greenfield.

The first main result on $C R$-submanifolds was obtained by Chen [2]: any $C R$ submanifold of a Kaehler manifold is foliated by totally real submanifolds (i.e., the totally real subbundle is involutive).

As nontrivial examples of $C R$-submanifolds, we can mention the (real) hypersurfaces of Hermitian manifolds.

2. Kenmotsu manifolds and their submanifolds. Tanno [7] has classified, into three classes, the connected almost contact Riemannian manifolds whose automorphisms groups have the maximum dimensions:

(1) homogeneous normal contact Riemannian manifolds with constant $\phi$ holomorphic sectional curvature;

(2) global Riemannian products of a line or circle and a Kaehlerian space form;

(3) warped product spaces $L \times_{f} F$, where $L$ is a line and $F$ a Kaehlerian manifold. 
Kenmotsu [5] studied the third class and characterized it by tensor equations. Later, such a manifold was called a Kenmotsu manifold.

A $(2 m+1)$-dimensional Riemannian manifold $(\tilde{M}, g)$ is said to be a Kenmotsu manifold if it admits an endomorphism $\phi$ of its tangent bundle $T \tilde{M}$, a vector field $\xi$, and a 1 -form $\eta$, which satisfy:

$$
\begin{gathered}
\phi^{2}=-\mathrm{Id}+\eta \otimes \xi, \quad \eta(\xi)=1, \phi \xi=0, \eta \circ \phi=0, \\
g(\phi X, \phi Y)=g(X, Y)-\eta(X) \eta(Y), \quad \eta(X)=g(X, \xi), \\
\left(\tilde{\nabla}_{X} \phi\right) Y=-g(X, \phi Y) \xi-\eta(Y) \phi X, \\
\tilde{\nabla}_{X} \xi=X-\eta(X),
\end{gathered}
$$

for any vector fields $X, Y$ on $\tilde{M}$, where $\tilde{\nabla}$ denotes the Riemannian connection with respect to $g$.

We denote by $\omega$ the fundamental 2-form of $\tilde{M}$, that is, $\omega(X, Y)=g(\phi X, Y)$, for all $X, Y \in \Gamma(T \tilde{M})$. It was proved that the pairing $(\omega, \eta)$ defines a locally conformal cosymplectic structure, that is,

$$
d \omega=2 \omega \wedge \eta, \quad d \eta=0 .
$$

A Kenmotsu manifold with constant $\phi$-holomorphic sectional curvature $c$ is called a Kenmotsu space form and it is denoted by $\tilde{M}(c)$. Then its curvature tensor $\tilde{R}$ is expressed by (cf. [5])

$$
\begin{aligned}
4 \tilde{R}(X, Y) Z=(c-3)\{ & g(Y, Z) X-g(X, Z) Y\} \\
+(c+1)[\{ & \{(X) Y-\eta(Y) X\} \eta(Z)+\{g(X, Z) \eta(Y)-g(Y, Z) \eta(X)\} \xi \\
& +\omega(Y, Z) \phi X-\omega(X, Z) \phi Y-2 \omega(X, Y) \phi Z] .
\end{aligned}
$$

Let $\tilde{M}$ be a Kenmotsu manifold and $M$ an $n$-dimensional submanifold tangent to $\xi$. For any vector field $X$ tangent to $M$, we put

$$
\phi X=P X+F X
$$

where $P X$ (resp., $F X$ ) denotes the tangential (resp., normal) component of $\phi X$. Then $P$ is an endomorphism of tangent bundle $T M$ and $F$ is a normal bundle valued 1-form on $T M$.

The equation of Gauss is given by

$$
\tilde{R}(X, Y, Z, W)=R(X, Y, Z, W)+g(h(X, W), h(Y, Z))-g(h(X, Z), h(Y, W)),
$$

for any vectors $X, Y, Z, W$ tangent to $M$.

We denote by $H$ the mean curvature vector, that is,

$$
H(p)=\frac{1}{n} \sum_{i=1}^{n} h\left(e_{i}, e_{i}\right),
$$

where $\left\{e_{1}, \ldots, e_{n}\right\}$ is an orthonormal basis of the tangent space $T_{p} M, p \in M$. 
Also, we set

$$
\begin{aligned}
h_{i j}^{r} & =g\left(h\left(e_{i}, e_{j}\right), e_{r}\right), \\
\|h\|^{2} & =\sum_{i, j=1}^{n} g\left(h\left(e_{i}, e_{j}\right), h\left(e_{i}, e_{j}\right)\right) .
\end{aligned}
$$

Let $\left\{e_{1}, \ldots, e_{n}\right\}$ be an orthonormal basis of $T_{p} M$. We put

$$
\|P\|^{2}=\sum_{i, j=1}^{n} g^{2}\left(P e_{i}, e_{j}\right) .
$$

By analogy with submanifolds in a Kaehler manifold, different classes of submanifolds in a Kenmotsu manifold were considered (cf. [6]).

A submanifold $M$ tangent to $\xi$ is said to be invariant (resp., anti-invariant) if $\phi\left(T_{p} M\right)$ $\subset T_{p} M$, for all $p \in M$ (resp., $\phi\left(T_{p} M\right) \subset T_{p}^{\perp} M$, for all $p \in M$ ).

A submanifold $M$ tangent to $\xi$ is called a contact $C R$-submanifold [9] if there exists a pair of orthogonal differentiable distributions $\mathscr{D}$ and $\mathscr{D}^{\perp}$ on $M$, such that,

(i) $T M=\mathscr{D} \oplus \mathscr{D}^{\perp} \oplus\{\xi\}$, where $\{\xi\}$ is the 1-dimensional distribution spanned by $\xi$;

(ii) $\mathscr{D}$ is invariant by $\phi$, that is, $\phi\left(\mathscr{D}_{p}\right) \subset \mathscr{D}_{p}$, for all $p \in M$;

(iii) $\mathscr{D}^{\perp}$ is anti-invariant by $\phi$, that is, $\phi\left(\mathscr{D}_{p}^{\perp}\right) \subset T_{p}^{\perp} M$, for all $p \in M$.

In particular, if $\mathscr{D}^{\perp}=\{0\}$ (resp., $\mathscr{D}=\{0\}$ ), $M$ is an invariant (resp., anti-invariant) submanifold.

Next, recall some notions introduced by Chen (see [3]).

Let $L$ be a $k$-plane section of $T_{p} M$ and $X$ a unit vector in $L$. We choose an orthonormal basis $\left\{e_{1}, \ldots, e_{k}\right\}$ of $L$ such that $e_{1}=X$.

Define the Ricci curvature $\operatorname{Ric}_{L}$ of $L$ at $X$ by

$$
\operatorname{Ric}_{L}(X)=K_{12}+K_{13}+\cdots+K_{1 k}
$$

where $K_{i j}$ denotes the sectional curvature of the 2-plane section spanned by $e_{i}, e_{j}$. We simply called such a curvature a $k$-Ricci curvature.

The scalar curvature $\tau$ of the $k$-plane section $L$ is given by

$$
\tau(L)=\sum_{1 \leq i<j \leq k} K_{i j}
$$

For each integer $k, 2 \leq k \leq n$, the Riemannian invariant $\Theta_{k}$ on an $n$-dimensional Riemannian manifold $M$ is defined by

$$
\Theta_{k}(p)=\frac{1}{k-1} \inf _{L, X} \operatorname{Ric}_{L}(X), \quad p \in M,
$$

where $L$ runs over all $k$-plane sections in $T_{p} M$ and $X$ runs over all unit vectors in $L$.

Recall that for a submanifold $M$ in a Riemannian manifold, the relative null space of $M$ at a point $p \in M$ is defined by

$$
\mathcal{N}_{p}=\left\{X \in T_{p} M \mid h(X, Y)=0, Y \in T_{p} M\right\} .
$$


3. Ricci curvature and squared mean curvature. Chen established a sharp relationship between the Ricci curvature and the squared mean curvature for submanifolds in real space forms (see [3]).

We prove similar inequalities for certain submanifolds of a Kenmotsu space form $\tilde{M}(c)$. We will consider submanifolds $M$ tangent to the Reeb vector field $\xi$.

THEOREM 3.1. Let $\tilde{M}(c)$ be a $(2 m+1)$-dimensional Kenmotsu space form and $M$ an $n$-dimensional submanifold tangent to $\xi$. Then

(i) for each unit vector $X \in T_{p} M$ orthogonal to $\xi$,

$$
\operatorname{Ric}(X) \leq \frac{1}{4}\left\{(n-1)(c-3)+\frac{1}{2}\left(3\|P X\|^{2}-2\right)(c+1)+n^{2}\|H\|^{2}\right\} ;
$$

(ii) if $H(p)=0$, then a unit tangent vector $X \in T_{p} M$ orthogonal to $\xi$ satisfies the equality case of (3.1) if and only if $X \in \mathcal{N}_{p}$;

(iii) the equality case of (3.1) holds identically for all unit tangent vectors orthogonal to $\xi$ at $p$ if and only if $p$ is a totally geodesic point.

Proof. Let $X \in T_{p} M$ be a unit tangent vector $X$ at $p$. We choose an orthonormal basis $e_{1}, \ldots, e_{n}=\xi, e_{n+1}, \ldots, e_{2 m+1}$ in $T_{p} \tilde{M}(c)$ such that $e_{1}, \ldots, e_{n}$ are tangent to $M$ at $p$, with $e_{1}=X$.

Then, from the equation of Gauss, we have

$$
n^{2}\|H\|^{2}=2 \tau+\|h\|^{2}-n(n-1) \frac{c-3}{4}-\left(3\|P\|^{2}-2 n+2\right) \frac{c+1}{4} .
$$

From (3.2), we get

$$
\begin{aligned}
n^{2}\|H\|^{2}= & 2 \tau+\sum_{r=n+1}^{2 m+1}\left[\left(h_{11}^{r}\right)^{2}+\left(h_{22}^{r}+\cdots+h_{n n}^{r}\right)^{2}+2 \sum_{i<j}\left(h_{i j}^{r}\right)^{2}\right] \\
& -2 \sum_{r=n+1}^{2 m+1} \sum_{2 \leq i<j \leq n} h_{i i}^{r} h_{j j}^{r}-n(n-1) \frac{c-3}{4}-\left(3\|P\|^{2}-2 n+2\right) \frac{c+1}{4} \\
= & 2 \tau+\frac{1}{2} \sum_{r=n+1}^{2 m+1}\left[\left(h_{11}^{r}+\cdots+h_{n n}^{r}\right)^{2}+\left(h_{11}^{r}-h_{22}^{r}-\cdots-h_{n n}^{r}\right)^{2}\right] \\
& +2 \sum_{r=n+1}^{2 m+1} \sum_{i<j}\left(h_{i j}^{r}\right)^{2}-2 \sum_{r=n+1}^{2 m+1} \sum_{2 \leq i<j \leq n} h_{i i}^{r} h_{j j}^{r} \\
& -n(n-1) \frac{c-3}{4}-\left(3\|P\|^{2}-2 n+2\right) \frac{c+1}{4} .
\end{aligned}
$$

From the equation of Gauss, we find

$$
\begin{aligned}
\sum_{2 \leq i<j \leq n} K_{i j}= & \sum_{r=n+1}^{2 m+1} \sum_{2 \leq i<j \leq n}\left[h_{i i}^{r} h_{j j}^{r}-\left(h_{i j}^{r}\right)^{2}\right] \\
& +\frac{(n-1)(n-2)}{2} \frac{c-3}{4} \\
& +\left(3\|P\|^{2}-3\left\|P e_{1}\right\|^{2}-2 n+4\right) \frac{c+1}{8} .
\end{aligned}
$$


Substituting (3.4) in (3.3), we get

$$
\begin{aligned}
\frac{1}{2} n^{2}\|H\|^{2}= & 2 \operatorname{Ric}(X)+\frac{1}{2} \sum_{r=n+1}^{2 m+1}\left(h_{11}^{r}-h_{22}^{r}-\cdots-h_{n n}^{r}\right)^{2} \\
& +\sum_{r=n+1}^{2 m+1} \sum_{j=1}^{n}\left(h_{1 j}^{r}\right)^{2}-2(n-1) \frac{c-3}{4}-\left(3\|P X\|^{2}-2\right) \frac{c+1}{4} \\
\geq & 2 \operatorname{Ric}(X)-2(n-1) \frac{c-3}{4}-\left(3\|P X\|^{2}-2\right) \frac{c+1}{4},
\end{aligned}
$$

which is equivalent to (3.1).

For (ii) assume that $H(p)=0$. Equality holds in (3.1) if and only if

$$
h_{12}^{r}=\cdots=h_{1 n}^{r}=0, \quad h_{11}^{r}=h_{22}^{r}+\cdots+h_{n n}^{r}=0, \quad r \in\{n+1, \ldots, 2 m\} .
$$

Then $h_{1 j}^{r}=0$, for all $j \in\{1, \ldots, n\}, r \in\{n+1, \ldots, 2 m\}$, that is, $X \in \mathcal{N}_{p}$.

For (iii) the equality case of (3.1) holds for all unit tangent vectors at $p$ if and only if

$$
\begin{gathered}
h_{i j}^{r}=0, \quad i \neq j, r \in\{n+1, \ldots, 2 m\}, \\
h_{11}^{r}+h_{22}^{r}+\cdots+h_{n n}^{r}-2 h_{i i}^{r}=0, \quad i \in\{1, \ldots, n\}, r \in\{n+1, \ldots, 2 m\} .
\end{gathered}
$$

It follows that $p$ is a totally geodesic point.

The converse is trivial.

COROLLARY 3.2. Let $M$ be an n-dimensional invariant submanifold tangent to $\xi$ in a Kenmotsu space form $\tilde{M}(c)$. Then,

(i) for each unit vector $X \in T_{p} M$ orthogonal to $\xi$, we have

$$
\operatorname{Ric}(X) \leq \frac{1}{4}\left\{(n-1)(c-3)+\frac{1}{2}(c+1)\right\} ;
$$

(ii) a unit tangent vector $X \in T_{p} M$ orthogonal to $\xi$ satisfies the equality case of (3.8) if and only if $X \in \mathcal{N}_{p}$

(iii) the equality case of (3.8) holds identically for all unit tangent vectors orthogonal to $\xi$ at $p$ if and only if $p$ is a totally geodesic point.

PROoF. It is known that every invariant submanifold of a Kenmotsu space form is minimal (cf. [6]).

On the other hand, for any unit tangent vector $X \in T_{p} M$ orthogonal to $\xi$, we have $\|P X\|=\|\phi X\|=\|X\|=1$.

Then, the inequality (3.1) implies (3.8).

Similarly, we can prove the following results.

COROLlary 3.3. Let $M$ be an n-dimensional anti-invariant submanifold tangent to $\xi$ in a Kenmotsu space form $\tilde{M}(c)$. Then,

(i) for each unit vector $X \in T_{p} M$ orthogonal to $\xi$, we have

$$
\operatorname{Ric}(X) \leq \frac{1}{4}\left\{(n-1)(c-3)-(c+1)+n^{2}\|H\|^{2}\right\} ;
$$


(ii) if $H(p)=0$, then a unit tangent vector $X \in T_{p} M$ orthogonal to $\xi$ satisfies the equality case of (3.9) if and only if $X \in \mathcal{N}_{p}$;

(iii) the equality case of (3.9) holds identically for all unit tangent vectors orthogonal to $\xi$ at $p$ if and only if $p$ is a totally geodesic point.

COROLLARY 3.4. Let $M$ be an n-dimensional contact $C R$-submanifold of a Kenmotsu space form $\tilde{M}(c)$. Then

(i) for each unit vector $X \in \mathscr{D}_{p}$,

$$
\operatorname{Ric}(X) \leq \frac{1}{4}\left\{(n-1)(c-3)+\frac{1}{2}(c+1)+n^{2}\|H\|^{2}\right\} ;
$$

(ii) for each unit vector $X \in \mathscr{D}_{p}^{\perp}$, we have

$$
\operatorname{Ric}(X) \leq \frac{1}{4}\left\{(n-1)(c-3)-(c+1)+n^{2}\|H\|^{2}\right\} .
$$

4. $k$-Ricci curvature. In this section, we prove a relationship between the $k$-Ricci curvature and the squared mean curvature for submanifolds tangent to $\xi$ in a Kenmotsu space form.

THEOREM 4.1. Let $\tilde{M}(c)$ be a Kenmotsu space form and $M$ an $n$-dimensional submanifold tangent to $\xi$. Then we have

$$
\|H\|^{2} \geq \frac{2 \tau}{n(n-1)}-\frac{c-3}{4}-\left(\frac{3\|P X\|^{2}-2(n-1)}{n(n-1)}\right) \frac{c+1}{4} .
$$

Proof. We choose an orthonormal basis $\left\{e_{1}, \ldots, e_{n}, e_{n+1}, \ldots, e_{2 m+1}=\xi\right\}$ at $p$ such that $e_{n+1}$ is parallel to the mean curvature vector $H(p)$, and $e_{1}, \ldots, e_{n}$ diagonalize the shape operator $A_{n+1}$. Then the shape operators take the forms

$$
\begin{gathered}
A_{n+1}=\left(\begin{array}{ccccc}
a_{1} & 0 & 0 & \cdots & 0 \\
0 & a_{2} & 0 & \cdots & 0 \\
\vdots & \vdots & \vdots & \ldots & \vdots \\
0 & 0 & 0 & \cdots & a_{n}
\end{array}\right) \\
A_{r}=\left(h_{i j}^{r}\right), \quad i, j=1, \ldots, n ; r=n+2, \ldots, 2 m, \quad \operatorname{trace} A_{r}=\sum_{i=1}^{n} h_{i i}^{r}=0 .
\end{gathered}
$$

From (3.2), we get

$$
\begin{aligned}
n^{2}\|H\|^{2}= & 2 \tau+\sum_{i=1}^{n} a_{i}^{2}+\sum_{r=n+2}^{2 m} \sum_{i, j=1}^{n}\left(h_{i j}^{r}\right)^{2} \\
& -n(n-1) \frac{c-3}{4}-\left(3\|P X\|^{2}-2(n-1)\right) \frac{c+1}{4} .
\end{aligned}
$$

On the other hand, since

$$
0 \leq \sum_{i<j}\left(a_{i}-a_{j}\right)^{2}=(n-1) \sum_{i} a_{i}^{2}-2 \sum_{i<j} a_{i} a_{j},
$$


we obtain

$$
n^{2}\|H\|^{2}=\left(\sum_{i=1}^{n} a_{i}\right)^{2}=\sum_{i=1}^{n} a_{i}^{2}+2 \sum_{i<j} a_{i} a_{j} \leq n \sum_{i=1}^{n} a_{i}^{2},
$$

which implies that

$$
\sum_{i=1}^{n} a_{i}^{2} \geq n\|H\|^{2}
$$

We have from (4.3)

$$
n^{2}\|H\|^{2} \geq 2 \tau+n\|H\|^{2}-n(n-1) \frac{c+3}{4}-\left(3\|P X\|^{2}-2(n-1)\right) \frac{c+1}{4},
$$

which is equivalent to (4.1).

Using Theorem 4.1, we obtain the following.

THEOREM 4.2. Let $\tilde{M}(c)$ be a Kenmotsu space form and $M$ an n-dimensional submanifold tangent to $\xi$. Then, for any integer $k, 2 \leq k \leq n$, and any point $p \in M$, we have

$$
\|H\|^{2}(p) \geq \Theta_{k}(p)-\frac{c-3}{4}-\frac{\left(3\|P\|^{2}-2 n+2\right)(c+1)}{4 n(n-1)} .
$$

Proof. Let $\left\{e_{1}, \ldots, e_{n}\right\}$ be an orthonormal basis of $T_{p} M$. Denote by $L_{i_{1}} \cdots i_{k}$ the $k$ plane section spanned by $e_{i_{1}}, \ldots, e_{i_{k}}$. It follows from (2.9) and (2.10) that

$$
\begin{aligned}
\tau\left(L_{i_{1} \cdots i_{k}}\right) & =\frac{1}{2} \sum_{i \in\left\{i_{1}, \ldots, i_{k}\right\}} \operatorname{Ric}_{L_{i_{1}} \cdots i_{k}}\left(e_{i}\right), \\
\tau(p) & =\frac{1}{C_{n-2}^{k-2}} \sum_{1 \leq i_{1}<\cdots<i_{k} \leq n} \tau\left(L_{i_{1} \cdots i_{k}}\right) .
\end{aligned}
$$

Combining (2.11) and (4.9), we find

$$
\tau(p) \geq \frac{n(n-1)}{2} \Theta_{k}(p) .
$$

From (4.1) and (4.10), we obtain (4.8).

In particular, we obtain the following.

COROLLARY 4.3. Let $M$ be an $n$-dimensional invariant submanifold tangent to $\xi$ in a Kenmotsu space form $\tilde{M}(c)$. Then, for any integer $k, 2 \leq k \leq n$, and any point $p \in M$,

$$
\Theta_{k}(p) \leq \frac{c-3}{4}+\frac{c+1}{4 n} .
$$

COROLlary 4.4. Let $M$ be an $n$-dimensional anti-invariant submanifold tangent to $\xi$ in a Kenmotsu space form $\tilde{M}(c)$. Then, for any integer $k, 2 \leq k \leq n$, and any point $p \in M$,

$$
\|H\|^{2}(p) \geq \Theta_{k}(p)-\frac{c-3}{4}+\frac{c+1}{2 n} .
$$


COROLlary 4.5. Let $M$ be an n-dimensional contact $C R$-submanifold of a Kenmotsu space form $\tilde{M}(c)$. Then, for any integer $k, 2 \leq k \leq n$, and any point $p \in M$,

$$
\|H\|^{2}(p) \geq \Theta_{k}(p)-\frac{c-3}{4}-\frac{(3 h-n+1)(c+1)}{2 n(n-1)},
$$

where $2 h=\operatorname{dim} \mathscr{D}$.

ACKNOWLEDGMENTS. The authors are very indebted to the referee for valuable suggestions. This paper is prepared during the third named author's visit to the Uludag University, Bursa, Turkey in July 2000. The third author is supported by the Scientific and Technical Research Council of Turkey (TÜBITAK) for NATO-PC Advanced Fellowships Programme.

\section{REFERENCES}

[1] D. E. Blair and B.-Y. Chen, On CR-submanifolds of Hermitian manifolds, Israel J. Math. 34 (1979), no. 4, 353-363.

[2] B.-Y. Chen, CR-submanifolds of a Kaehler manifold. I, J. Differential Geom. 16 (1981), no. 2, 305-322.

[3] _ Relations between Ricci curvature and shape operator for submanifolds with arbitrary codimensions, Glasgow Math. J. 41 (1999), no. 1, 33-41.

[4] B.-Y. Chen and K. Ogiue, On totally real submanifolds, Trans. Amer. Math. Soc. 193 (1974), 257-266.

[5] K. Kenmotsu, A class of almost contact Riemannian manifolds, Tôhoku Math. J. (2) 24 (1972), 93-103.

[6] K. Matsumoto, I. Mihai, and R. Roşca, A certain locally conformal almost cosymplectic manifold and its submanifolds, Tensor (N.S.) 51 (1992), no. 1, 91-102.

[7] S. Tanno, The automorphism groups of almost contact Riemannian manifolds, Tôhoku Math. J. (2) 21 (1969), 21-38.

[8] K. Yano and S. Ishihara, The $f$-structure induced on submanifolds of complex and almost complex spaces, Kōdai Math. Sem. Rep. 18 (1966), 120-160.

[9] K. Yano and M. Kon, Structures on Manifolds, Series in Pure Mathematics, vol. 3, World Scientific Publishing, Singapore, 1984.

KadRi ARsLan: Department of Mathematics, Faculty of ARTS AND SCiences, UludaG UNIVERSITY, GÖRÜKLE 16059, BURSA, TURKEY

E-mail address: ars1an@uludag.edu.tr

Ridvan Ezentas, Cengizhan Murathan, and Cihan Özgür: Department of MathematICS, FACUlTy OF ARTS AND SCIENCES, UludAG UniVERSiTy, GÖRÜKLE 16059, BURSA, TURKEY

Ion Mihai: Faculty of Mathematics, University of Bucharest, Str. Academiei 14, 70109 BUCHAREST, ROMANIA

E-mail address: imihai@math . math. unibuc. ro 


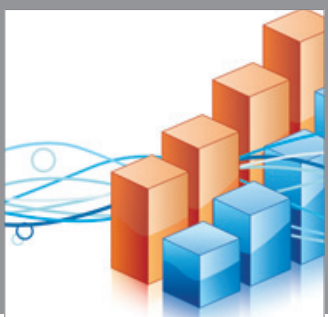

Advances in

Operations Research

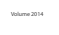

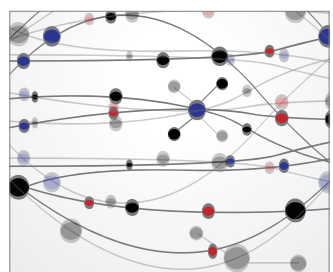

\section{The Scientific} World Journal
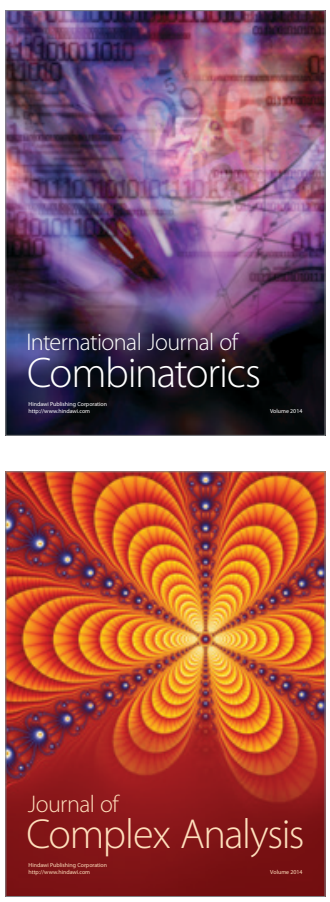

International Journal of

Mathematics and

Mathematical

Sciences
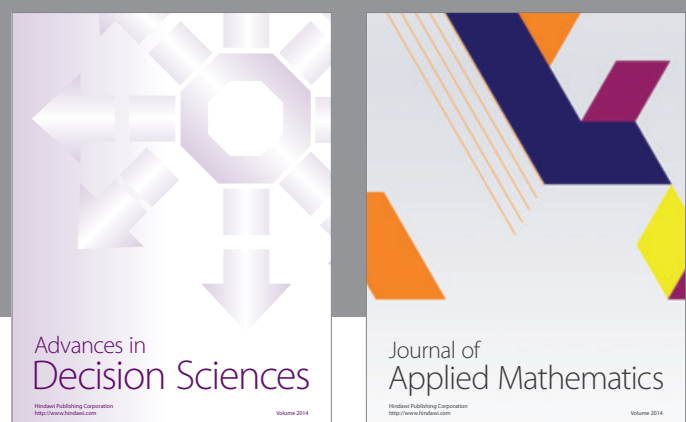

Journal of

Applied Mathematics
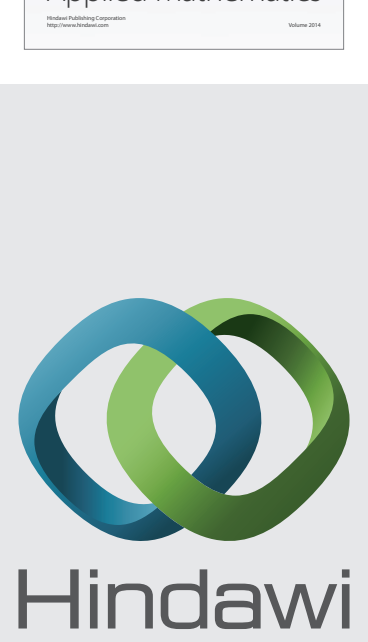

Submit your manuscripts at http://www.hindawi.com
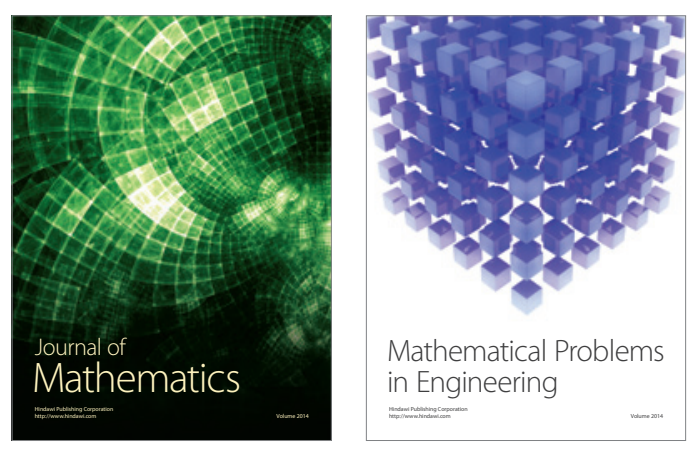

Mathematical Problems in Engineering
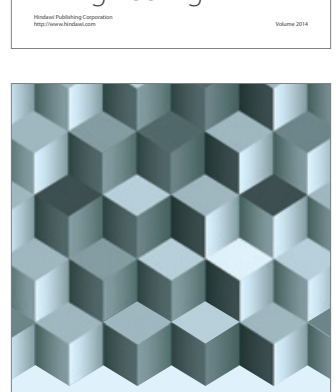

Journal of

Function Spaces
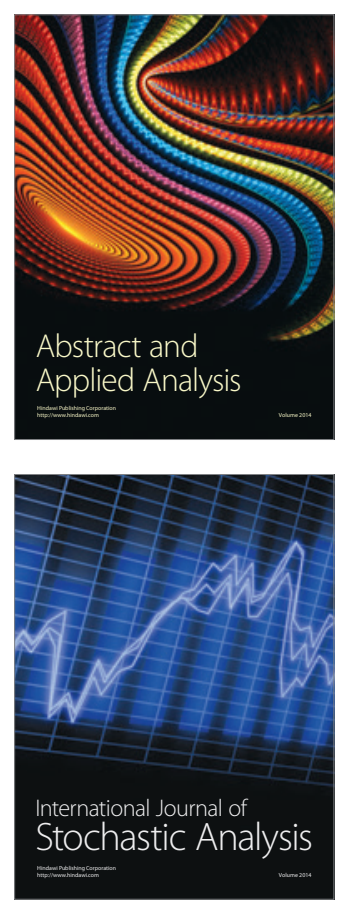

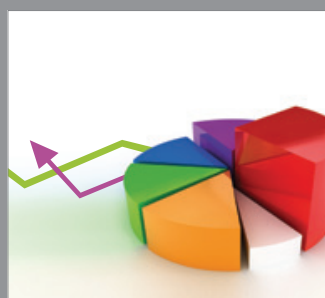

ournal of

Probability and Statistics

Promensencen
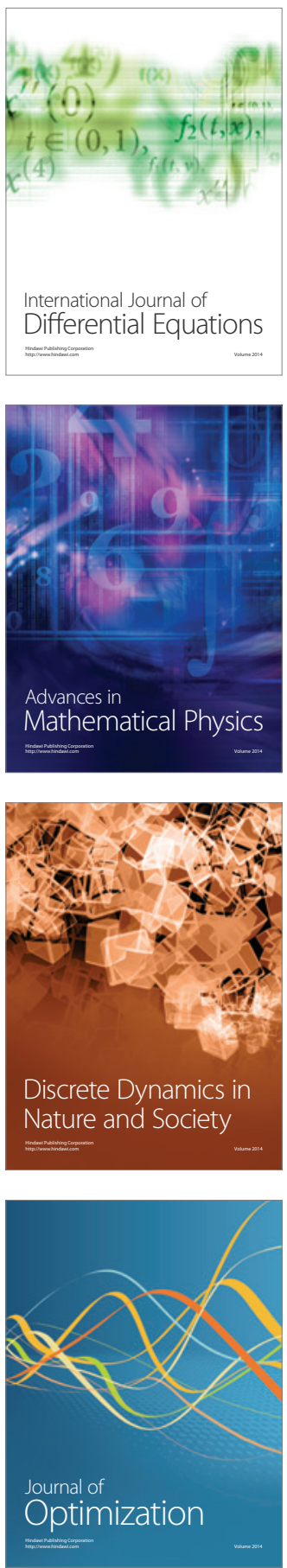